\title{
Study on the flow field in the hydrocyclone with double conical structure
}

\author{
Bo Liu ${ }^{1,2, a}$, Juan $\mathrm{Xu}{ }^{1,2, b}$ and Yue Wang ${ }^{1,2, \mathrm{c}}$ \\ ${ }^{1}$ Shandong Provincial Key Laboratory of Ocean Environment Monitoring Technology, Qingdao \\ 266001, China \\ ${ }^{2}$ Institute of Oceanographic Instrumentation, Shandong Academy of Sciences, Qingdao 266100, \\ China. \\ ahanyu_8224@126.com, bxujuan101066@163.com, 'shinjiwy @163.com
}

Keywords: hydrocyclone, the structure parameter, numerical simulation, large eddy simulation.

\begin{abstract}
The large eddy simulation method is used to simulate the flow field in the hydrocyclone with double conical structure, and the result shows that existence of the secondary swirl has a vital effect on the separation. The tangential velocity in the swirling flow could be regarded as a kind of rankine-like vortex. And the gradient near the axis is large. At the same time two-way velocity gradient is significantly larger. Velocity near the wall gradually decreases. The flow field of random fluctuation affects the oil column size and its stability. This instability in turn intensifies the turbulent in flow field, which leads to the reduction of the separating efficiency.
\end{abstract}

\section{Introduction}

Cyclone is an efficient separation device, with simple structure, high separation efficiency, flexible operation and many other advantages. And it is widely used in coal, petroleum, chemical industry, food industry, waste water treatment, etc. With the wide application of computers and the increasing storage capacity, the simulation has good accuracy and reliability. In recent years, it is possible to simulate the internals of separation equipment, transportation equipment and reaction equipment. And the calculated results agree well with the test results [1-5]. In this paper numerical simulation method is used to analyze the flow of the internal flow field in the hydrocyclone with double conical structure. And the results provides the basis for further study of separation efficiency and the changing rule of the energy consumption in the process of oil-water separation hydrocyclone.

\section{Fluid Model}

Large eddy simulation control equation is the averaged equation after filtering the N-S equation.

$$
\frac{\partial \overline{\mathrm{u}_{i}}}{\partial t}+\frac{\partial}{\partial x_{j}}\left(u_{i} u_{j}\right)=-\frac{1}{\rho} \frac{\partial \bar{p}}{\partial x_{i}}+v \frac{\partial^{2} \overline{u_{i}}}{\partial x_{j}^{2}}+f_{i}
$$

The micro compressible flow field model is used in the continuity equation.

$$
\frac{\partial P}{\partial t}+K \frac{\partial \overline{u_{i}}}{\partial x_{i}}=0
$$

In the above equation, horizontal line presents the quantity after filtering. Relation between the filtration rate $\bar{u}_{i}$ and actual speed $u_{i}$ in the large eddy model is $u=\overline{u_{i}}+u_{i}^{\prime}$. K is the square of fluid elastic wave velocity. Nonlinear term exits in the momentum equation. $\overline{u_{i} u_{j}}=\overline{\overline{u_{i} u_{j}}}+\overline{u_{i}^{\prime} \overline{u_{j}}}+\overline{\overline{u_{i} u_{i}^{\prime}}}+\overline{u_{i}^{\prime} u_{j}^{\prime}}$. The first item is large scale component and the other three items are small scale components, that is sub-grid reynolds stress. Then it can be expressed as $\overline{u_{i} u_{j}}=\overline{\overline{u_{i} u_{j}}}+R_{i j} . R_{i j}$ is the new unknown after filtering, so sub-grid reynolds stress model is established. Then box-filter function is used and the equation (1) can be expressed as follows. 


$$
\frac{\partial \overline{\mathrm{u}_{i}}}{\partial t}+\frac{\partial}{\partial x_{j}}\left(\overline{u_{i} u_{j}}\right)=-\frac{1}{\rho} \frac{\partial \bar{p}}{\partial x_{i}}+v_{t} \frac{\partial^{2} \overline{u_{i}}}{\partial x_{j}^{2}}-\frac{\partial \tau_{i j}}{\partial x_{j}}+f_{i}
$$

According to the average concept of the turbulence energy generated and dissipation, Smagorinsky assumes $v_{\mathrm{t}}=\left(C_{s} \Delta\right)^{2}\left(2 \overline{S_{i j}} \bar{S}_{i j}\right)^{1 / 2}$ and $\overline{S_{i j}}=\frac{1}{2}\left[\frac{\partial \overline{u_{i}}}{\partial x_{j}}+\frac{\partial \overline{u_{i}}}{\partial x_{i}}\right] . \Delta$ is filter width, generally it is grid spacing and $\Delta=\left(\Delta_{x} \Delta_{y} \Delta_{z}\right)^{1 / 3}$. $C_{s}$ is not only the model coefficient but also empirical constant. When it is taken as 0.1 the result consistent with the experiment can be obtained. So in this paper $C_{s}$ is 0.1 .

\section{Calculation model and boundary conditions}

The calculation model of the hydrocyclone with double conical structure is shown in Fig.1. The diameter of whirl cavity is $2 \mathrm{D}$, the length is $2 \mathrm{D}$, inlet diameter is $0.35 \mathrm{D}$ and the taper is $2.5^{\circ}$.

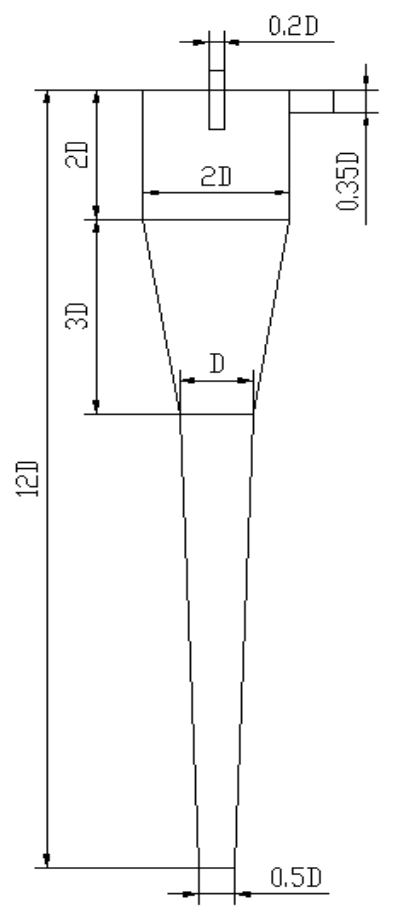

Fig. 1 Structure diagram of the hydrocyclone

The equation is discrete by the finite volume method and SIMPLE algorithm is used for the pressure-velocity coupling. The wall is not leaking with non slip condition. Shear, turbulent kinetic energy and turbulent diffusivity are calculated by wall function. The unstructured grid is used to mesh the calculation area. The inlet velocities are $1 \mathrm{~m} / \mathrm{s}, 3 \mathrm{~m} / \mathrm{s}$ and $5 \mathrm{~m} / \mathrm{s}$.

\section{The discussion and analysis}

Different tangential velocity distributions in the inner regions of the cyclone are shown in Fig.2. It can be seen that the changes of the tangential velocity are small in outer cyclone. Its size changes are fluctuations, and there is not much change in direction. While in inner cyclone the size and direction of the tangential velocity change greatly.

The longitudinal velocity distributions in cylinder - cone region and central region of the cone are in Fig.3. Although longitudinal velocity increases with the decrease of the radius from the wall to the center, there are irregular changes in different position of the axis. That is there is the asymmetric distribution. It shows secondary vortex is in this region. The strong radial turbulence fluctuation of secondary vortex causes radial velocity fluctuating changes in size and direction, which illustrates that secondary vortex area has a great influence on separation. 


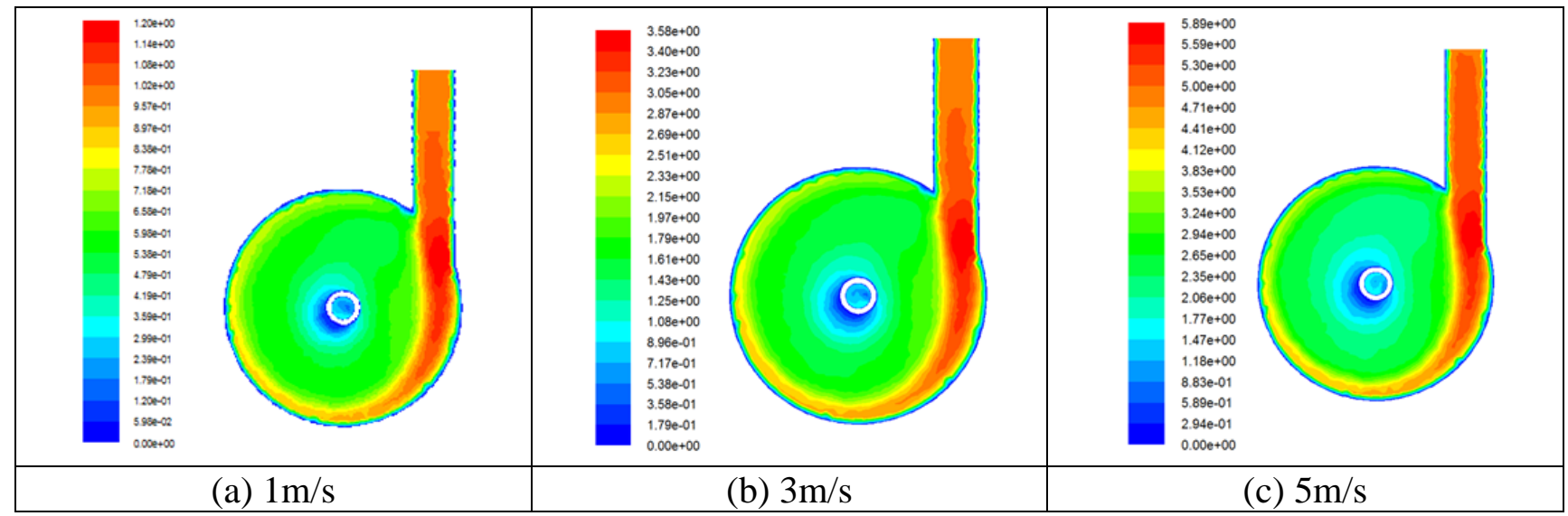

Fig 2 Different tangential velocity distributions

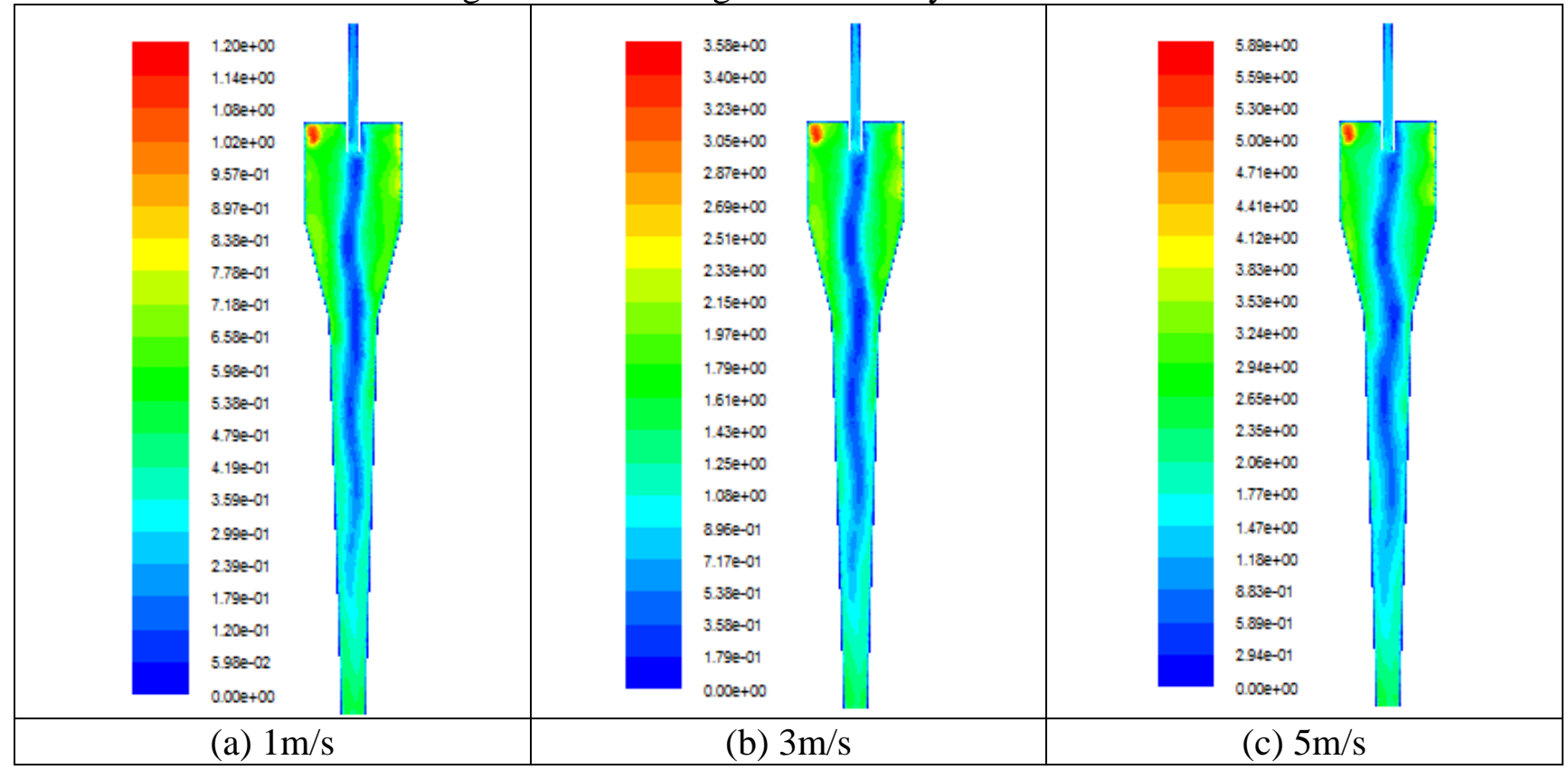

Fig.3 Different tangential velocity distributions

The tangential velocity in the swirling flow could be regarded as a kind of rankine-like vortex. And the gradient near the axis is large. At the same time two-way velocity gradient is significantly larger. Velocity near the wall gradually decreases. In the swirling flow along the axial direction, there are two areas flowing in opposite directions. From the axis to the wall, the axial velocity changes from negative to positive. The points whose axial velocity is zero form the zero axial velocity envelope. And its inclined direction is in the same direction of the cone. The inner fluid flows upward to form inner vortex and the outer fluid flows downward to from the outer vortex. With the constraint of mass conservation, axial velocity, radial velocity and tangential velocity are interrelated. While in the swirling flow the radial velocity is smaller than the tangential velocity and axial velocity. Then any small changes of axial velocity and tangential velocity can cause the great change of the radial velocity. Therefore, numerical simulation result of the radial velocity along the radial distribution is more complex.

The flow field of random fluctuation affects the oil column size and its stability. This instability in turn intensifies the turbulent in flow field, which leads to the reduction of the separating efficiency. Pressure distributions in the hydrocyclone are shown in Fig.4. It shows that the longitudinal pressure gradient gradually increases. There is positive pressure gradient near the axis and negative pressure gradient near the wall. The two opposite axial gradients are in the swirling flow, then inner vortex is formed along the axis. 


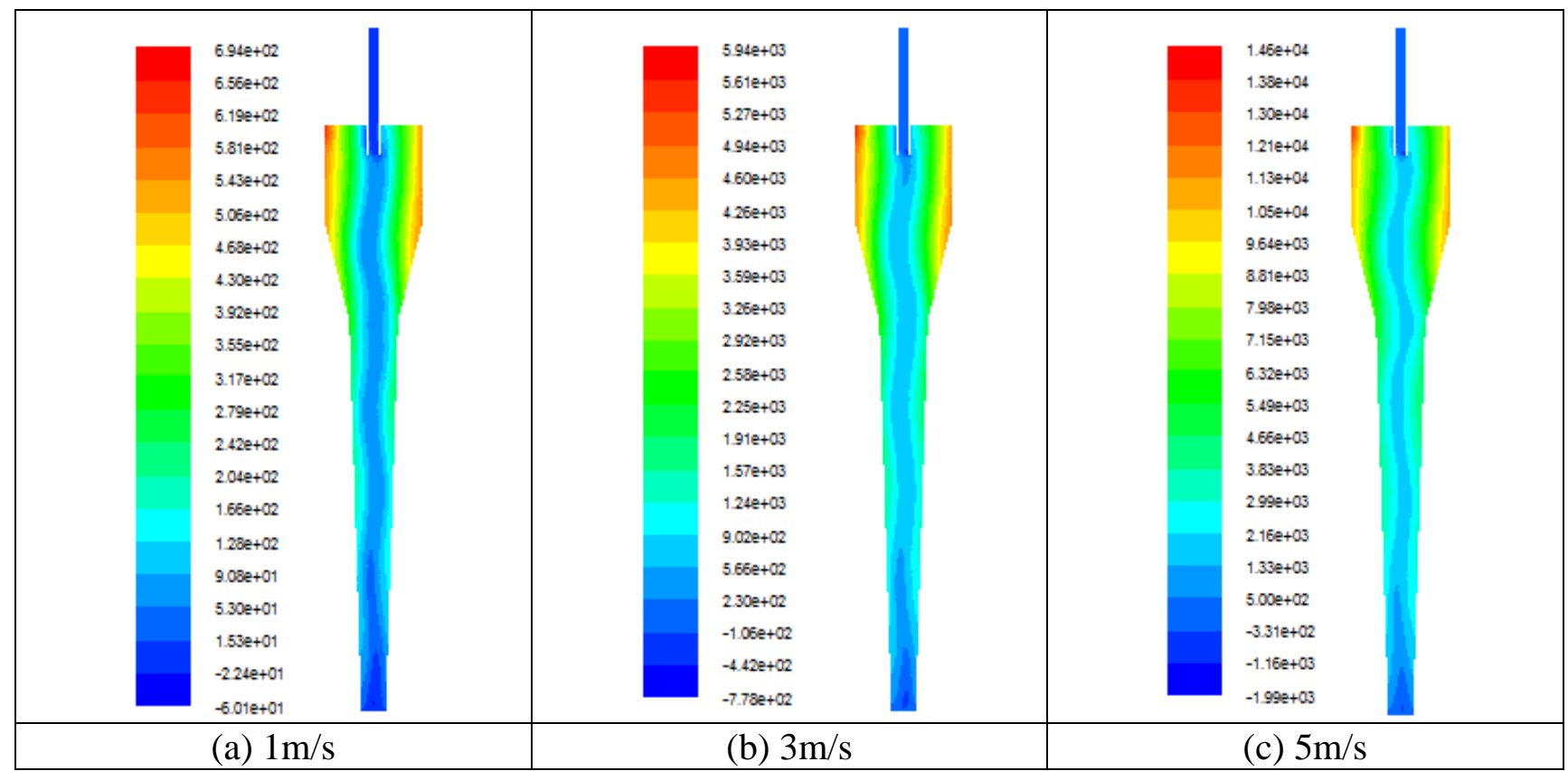

Fig.4 Pressure distributions

\section{Conclusions}

The large eddy simulation method is used to simulate the flow field in the hydrocyclone with double conical structure, and the result shows that existence of the secondary swirl has a vital effect on the separation. The tangential velocity in the swirling flow could be regarded as a kind of rankine-like vortex. And the gradient near the axis is large. At the same time two-way velocity gradient is significantly larger. Velocity near the wall gradually decreases. The flow field of random fluctuation affects the oil column size and its stability. This instability in turn intensifies the turbulent in flow field, which leads to the reduction of the separating efficiency.

\section{Acknowledgements}

This work was financially supported by the Youth Foundation of Shandong Academy of Sciences (Project No. 2014QN033).

\section{References}

[1]. Cullivan J. C., et al., New understanding of a hydrocyclone flow field and separation mechanism from computational fluid dynamics, Minerals Engineering Vol. 17(2004): p. 651-660.

[2]. Li Jianming. Numerical simulation of turbulence two-phase flow within hydrocyclone and their separation performance [D]. Chengdu:Sichuan Unit Univ.,1997.

[3]. Jiang Minghu, Li Feng, et al., Radial Pressure Field Study of Hydrocyclones, Journal of Daqing Pe troleum Institute, 23(1), 1999, pp60-62.

[4]. Wang Zunce, Gao Ye, et al., Effect of Liquid-liquid Hydrocyclone's Inlet Structure on Pressure Characteristic, 31(2), 2003, pp16-19.

[5]. Zhao Qingguo, The Development and Appl ication of Liquid-Liquid Hydrocyclone, Chemical Industry and Engineering, 17(3), 2000, pp141-148 